\title{
Haptic Rendering of Cultural Heritage Objects at Different Scales
}

\author{
Sreeni K. G., Priyadarshini K., Praseedha A. K., and Subhasis Chaudhuri * \\ Vision and Image Processing Laboratory, Department of Electrical Engineering, \\ Indian Institute of Technology Bombay, Powai, Mumbai-400076 \\ \{sreenikg, pkumari, praseedha, sc\}@ee.iitb.ac.in
}

\begin{abstract}
In this work, we address the issue of virtual representation of objects of cultural heritage for haptic interaction. Our main focus is to provide a haptic access of artistic objects of any physical scale to the differently abled people. This is a low-cost system and, in conjunction with a stereoscopic visual display, gives a better immersive experience even to the sighted persons. To achieve this, we propose a simple multilevel, proxy-based hapto-visual rendering technique for point cloud data which includes the much desired scalability feature which enables the users to change the scale of the objects adaptively during the haptic interaction. For the proposed haptic rendering technique the proxy updation loop runs at a rate 100 times faster than the required haptic updation frequency of $1 \mathrm{KHz}$. We observe that this functionality augments very well to the realism of the experience.
\end{abstract}

Keywords: Haptic rendering, HIP, proxy-based rendering, voxel based rendering, image pyramid, virtual museum, stereoscopic display.

\section{Introduction}

In the recent years digital technology is paving a way into safeguarding cultural heritages, and it also offers a great promise for enhancing access to them. A user's experience of accessing such cultural objects can be made more realistic and immersive by incorporating the recently evolving haptic technologies. Museum of Pure Form [2], a virtual reality system placed inside several museums and art galleries around Europe is an attempt to use of haptic technologies in cultural heritage applications. The incorporation of haptics in cultural heritage applications also helps in letting visually impaired people feel the exhibits that are behind glass enclosures, making even very fragile objects available to the scholars and allowing museums to show off a range of artefacts that are currently in storage due to lack of space. Further a joint hapto-visual rendering improves the immersivenes of the kinesthetic interaction. Some existing systems also allow users to interact with museum exhibition pieces via the internet [16]. It is required that such a system should enable the users to hapto-visually explore ancient monuments and heritage sites like Taj Mahal. However, currently available haptic systems are unable to handle objects at different scales.

\footnotetext{
* This work was supported in part by a DST grant on Indian Digital Heritage and another by MCIT on perception engineering.
} 
As a part of our exercise in preserving our cultural heritage we propose a simple multilevel hapto-visual rendering technique with depth data of cultural heritage objects. With mesh models of objects there are effective rendering techniques in haptics like god object rendering algorithm as proposed in [17]. However this algorithm fails in the case of point cloud based models. Further cultural objects appear at various different scales, and the user needs to experience the object at different levels of details. A meshbased haptic technique is not amenable to scale changes as it requires the mesh to be pre-computed at all scales which is not feasible. In this paper, we propose a fast, proxy based rendering technique capable of working with point cloud based $3-D$ models. Additionally the proposed method is amenable to haptic rendering at various scales. In order to render the model at different levels of resolution, we generate depth at each point of the model by reading the contents of depthbuffer in OpenGL and create a Monge surface from it. We show that the user's experience can be improved by allowing the user to interact with the object at multiple resolutions. This feature allows the user to feel the object more precisely at a closer level when needed and zoom out when context is desired. We have also developed a graphical user interface to make accessibility easier. Moreover, the easy availability of $3-D$ models makes it a cost-effective system to savour the experience of various cultural heritage sites. The key contribution in this paper include how to render a Monge surface represented by a non-uniform point cloud data and how to handle scale change for zooming in and out during haptic interaction.

\section{Literature Review}

In the haptic rendering literature there are mainly two different approaches: Polygon (geometry) based rendering and Voxel based rendering. A good introduction to the basic haptic rendering technique is given by [7], [14]. Traditional haptic rendering method is based on a geometric surface representation which consists of mainly triangular or polygonal meshes. In polygon based rendering, each time the haptic interface point (HIP) penetrates the object, the haptic rendering algorithm calculates the closest surface point on the polygonal mesh and the corresponding penetration depth. If $\mathbf{d}$ is the vector representing the depth of penetration in the model, the reaction force can be calculated as $\mathbf{F}=-k \mathbf{d}$, where $k$ is the stiffness constant, a physical property of the associated surface. The above method has problems while determining the appropriate direction of the force while rendering thin objects. Zilles and Salisbury [17], and Ruspini et al. [13] independently introduced the concept of god-object and proxy algorithm, respectively, which can solve the problems associated with thin objects.

In the God-Object rendering method [17], the authors use a second point in addition to the HIP called "god-object", sometimes called the ideal haptic interface point (IHIP). While moving in free space the god-object and the HIP are collocated. However, as the HIP penetrates the virtual object, the god-object is constrained to lie on the surface of the virtual object. The position of the god-object can be determined by minimizing the energy of the spring between the god-object and the HIP taking into account constraints represented by the faces of the virtual object [7]. If $(x, y, z)$ are the coordinates of the proxy lying on the virtual object and $\left(x_{h}, y_{h}, z_{h}\right)$ represents the coordinates of the HIP, 
the spring energy is given by

$$
L=\frac{\left(x-x_{h}\right)^{2}}{2}+\frac{\left(y-y_{h}\right)^{2}}{2}+\frac{\left(z-z_{h}\right)^{2}}{2}+\sum_{i=1}^{3} l_{i}\left(A_{i} x+B_{i} y+C_{i} z-D_{i}\right)
$$

where $L$ is the cost function to be minimized, $l_{1}, l_{2}, l_{3}$ are Lagrange multipliers and $\left(A_{i}\right.$, $\left.B_{i}, C_{i}, D_{i}\right)$ are the homogeneous coefficients for the constraint plane equations on which the proxy lies. The 'force shading' technique (haptic equivalent of Phong shading) introduced by Morgenbesser and Srinivasan refined the above algorithm while rendering smooth objects [11]. Mesh based haptic rendering is not amenable to object scaling as the constraint equation for the planes $\left(A_{i}, B_{i}, C_{i}, D_{i}\right)$ must be recomputed.

Volume haptic rendering technique is another alternative rendering technique used in haptics. The most basic representation for a volume is the classic voxel array in which each discrete spatial location has a one-bit label indicating the presence or absence of material. Avila et al. have used additional physical properties like stiffness, color and density during the voxel representation [1]. The voxmap-point shell algorithm uses the voxel map for stationary objects and point shell for dynamic objects [10], [12]. Point shell has been defined as a set of point samples and associated inward facing normals. However, these normals are not available and one needs to compute the normal at every location. The external surface $\partial O$ of a solid object $O$ can be described by the implicit equation as [6]

$$
\partial O=\left\{(x, y, z) \in R^{3} \mid \phi(x, y, z)=0\right\},
$$

where $\phi$ is the implicit function (also called the potential function) and $(x, y, z)$ is the coordinate of a point in $3-D$. In other words, the set of points for which the potential is zero defines the implicit surface. This has found applications in haptic rendering. This technique also suffers from the thin object problem. Lee et al. have proposed a rendering technique with point cloud data which computes the distance from HIP to the closest point on the moving least square (MLS) surface defined by the given point set[8]. Here the same problem occurs as in the distance field based rendering technique, since we do not keep track of HIP penetration and therefore is not good in rendering thin objects. El-Far et al. used axis aligned bounding boxes to fill the voids in the point cloud and then rendered with a god object rendering technique[4]. Leeper et al. described a constraint based approach of rendering point cloud based data where the points are replaced by spheres or surface patches of approximate size [9]. Another proxy based technique of rendering dense 3D point cloud was proposed in [15], where the surface normal is estimated locally from the point cloud.

\section{Proposed Method}

The rendering technique we propose is a proxy based method which does not use polygonal meshes for the reasons mentioned earlier. In practice, most of the cultural objects are carefully preserved and a dense $3-D$ scan is performed on these objects to create virtual $3-D$ model in the form of .obj, .ply, .3ds file, etc. Instead we directly use the point cloud data defining the models. As mentioned in the introduction, we get the depth data in the form of $z_{i}=f\left(x_{i}, y_{i}\right)$ where $x_{i}$ and $y_{i}$ are discrete values and $z_{i}$ is the 
height of each sampled point from a reference plane $(z=0)$ and $i$ and $j$ can take values depending on the size of the model. We haptically render the sampled surface of the object approximated by the depth values. In order to haptically render the object, we need to find the collision of HIP with the bounding surface and hence the penetration depth of HIP into the surface. The key factors in haptic rendering algorithm are-

1. The magnitude of the haptic force should be proportional to the penetration depth of HIP from the surface.

2. The direction of force should be normal to the surface at the point of contact of the proxy.

By taking these factors into consideration, the proposed algorithm tries to move the proxy over the object surface in short steps during the interaction so that each time it finds the most appropriate proxy position, the new position minimizes the distance between HIP and proxy and at the same time applies the reaction force normal to the surface at the point of contact. Initially, let us assume that the surface is known or the values of $z$ is known for all values of $x$ and $y$ in continuum.

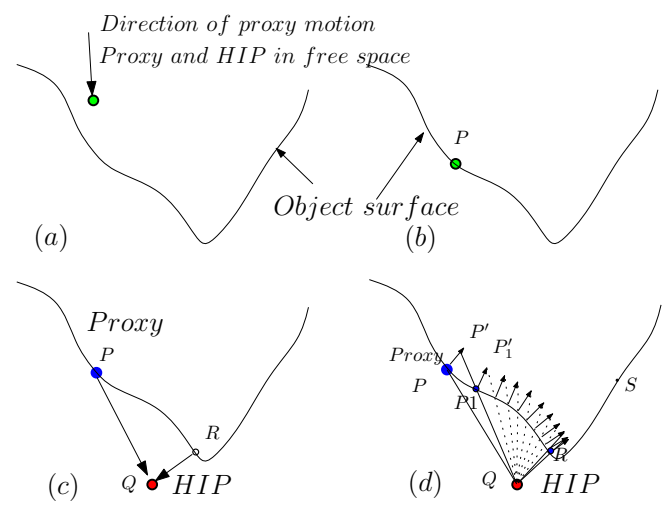

Fig. 1: Illustration of the proposed method to find the penetration depth of HIP into the surface.

To understand our procedure let us look at the situation in Fig. 1] The bounding surface of the object is shown with the curve. In free space, proxy and HIP are collocated and is shown with green circle above the surface. Let the HIP and the proxy be in free space at a time $t=t_{0}$ as shown in Fig. [1(a). The HIP and hence the proxy are together moving towards the surface in a direction as shown by the arrow. At $t=t_{1}$, let HIP and proxy touch the surface at point $P$ as in Fig. 1(b). Up to this point the proxy moves with the HIP. If the HIP is moved further in the direction it penetrates the surface and let $Q$ as shown in Fig. 1 ( c) be the HIP position at time $t=t_{2}$. Now the proposed algorithm finds the most appropriate position of proxy at $R$ where the distance $Q R$ is minimum, and the penetration depth $Q R$ is calculated in the direction exactly opposite to the surface normal at $R$. To find the point $R$ from the starting point $P$ we use the 
successive approximation method and move the proxy $P$ to a distance $\delta \mathbf{n}$ along the normal to the surface at point $P^{\prime}$ and draw a line $P^{\prime} Q$ to the current HIP as shown in Fig. 1. d). The point $P_{1}$ on the surface at which the line intersects is found and is updated as the current proxy position. Again we move the proxy point $P_{1}$ along the normal at the current proxy position to $P_{1}^{\prime}$ and the process is repeated until the final proxy position $R$ is attained. This is a greedy method but works well for smooth surfaces. If the surface has a fine texture, the line $P^{\prime} Q$ may intersect the surface at multiple points and the process may converge to a poor, local minima, yielding a jerky haptic interaction. In case of multiple intersections, the one closest to $P^{\prime}$ is selected. The length of the vector $\delta \mathbf{n}$ determines the rate of convergence of the process. A large value of $\delta \mathbf{n}$ may lead to some spurious interaction when $P P^{\prime}$ may intersect the surface along the segment $R S$ instead of $P R$. Hence a smaller value of $\delta \mathbf{n}(0.1 \mathrm{~mm}$ is used in our algorithm) is preferred for rendering purposes. Quite naturally, it is required that the proxy position updation is performed within $1 \mathrm{~ms}$ of time, so that the user's interaction with the object through the haptic device is unhindered and is carried at $1 \mathrm{KHz}$. Till now, we have assumed

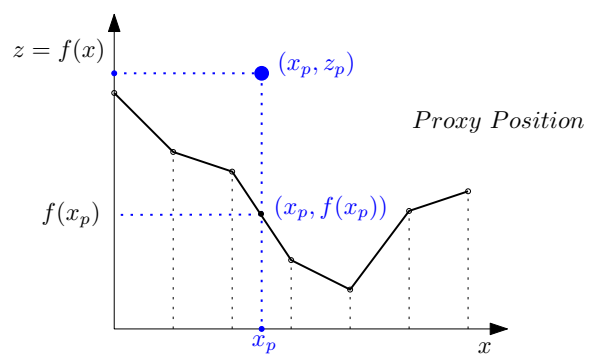

Fig. 2: Surface approximation from depth values.

the surface to be known. Now, we try to approximate the surface from the given depth values. In case of $2-D$ depth data, we project the proxy onto the X-Y plane and the corresponding depth value is obtained by interpolating the neighbourhood depth values to form a continuous function $z=f(x, y)$. For better understanding, we consider a onedimensional function $z=f(x)$ as shown in Fig. 2. As we have the function defined only at sampled points we interpolate the function at $x_{p}$ to find the value of $f\left(x_{p}\right)$. Since the available points are sampled quite densely, bilinear interpolation is sufficient to find the bounding surface as shown in Fig. 2. In order to check the collision of HIP with the function we perform the following. At a given proxy position $\left(x_{p}, z_{p}\right)$ we check for the function value $f\left(x_{p}\right)$. If $f\left(x_{p}\right)>z_{p}$ proxy has touched the surface, otherwise it is free to move towards the HIP. Extending the concept to $2-D$ depth data, let $\mathbf{X}_{h}=\left[x_{h}, y_{h}, z_{h}\right]^{T}$ denotes the HIP point and $\mathbf{X}_{p}=\left[x_{p}, y_{p}, z_{p}\right]^{T}$ denotes the proxy point. Collision can be easily checked here by comparing $z_{p}$ with the depth interpolated at the projected point 
$z=f(x, y)$. The proxy movement during the rendering is managed by equation 2 .

$$
\begin{aligned}
\mathbf{X}_{p}^{(k+1)} & =\mathbf{X}_{p}^{(k)}+\delta \mathbf{n} \quad \text { if } \quad z_{p}<f\left(x_{p}, y_{p}\right) \\
& =\mathbf{X}_{p}^{(k)}+|\delta \mathbf{n}| \frac{\left(\mathbf{X}_{h}-\mathbf{X}_{p}\right)^{(k)}}{\left|\mathbf{X}_{h}-\mathbf{X}_{p}\right|^{(k)}} \quad \text { otherwise }
\end{aligned}
$$

This allows a smooth interaction when the force is withdrawn out of the object. The updated proxy then slowly moves towards the new HIP position.

\section{Rendering}

Rendering part of our work concerns with both haptic rendering and graphic rendering. Haptic rendering involves generating software controlled forces and feeding it to the users to provide them the sensation of touch. Any haptic rendering technique must include two steps:

1. detection of collision of the HIP with the object.

2. force computation if a collision is detected.

If $z_{p}<f\left(x_{p}, y_{p}\right)$ then the proxy has touched the object and a force needs to be fed back by the haptic device. Subsequently, the reaction force is computed as $\mathbf{F}=-k \mathbf{d}$ where $k$ is the Hooke's constant, and $\mathbf{d}$ is the penetration depth given by $\mathbf{d}=\left|\mathbf{X}_{h}-\mathbf{X}_{p}\right|$, where $\mathbf{X}_{h}$ is the HIP position and $\mathbf{X}_{p}$ is the proxy position. Here we assume the stiffness to be constant everywhere on the surface of the object, but it can also be a function of position, provided the material property of the object is well documented. Fig. 3a shows proxy and HIP positions while rendering an arbitrary surface. For illustration we have selected only a small part of the depth map around the active region. The blue ball is the computed proxy touching the surface while the HIP is penetrated inside the surface. The HIP is shown with a red ball in the scene and the line from HIP to proxy is normal to the surface point at the proxy position. In order to show the surface of the object graphically

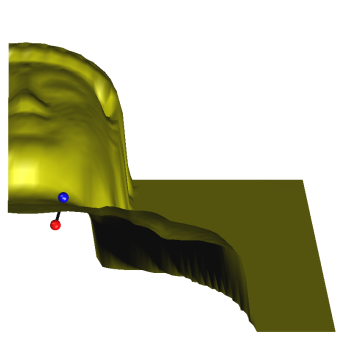

(a)

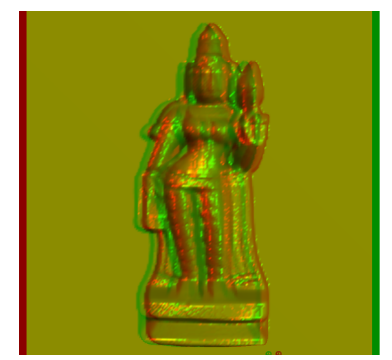

(b)

Fig. 3: (a) Illustration of proxy and HIP positions for an arbitrary surface. (b) Stereoscopic view of an Indian heritage object. (Data Courtesy: www.archibaseplanet.com) 
for simultaneous visual immersion, we display the image as a simple quad mesh out of the depth values. The normal is computed at each vertex. Although, we use point cloud data for haptic rendering, using the same for graphic rendering would result in gaps in the visually rendered object. Hence we have opted for the mesh-based graphical display in order to give a better perception to the viewer. We have used the stereoscopic display technique for creating the effect of depth in the image by presenting two offset images in different colours separately to the left and right eye of the viewer. A human observer combines these $2-D$ offset images to recreate the $3-D$ perception. Anaglyphic glasses can be used to filter offset images from a single source, separated to each eye to give the perception of a $3-D$ view to the users. Fig. $3 b$ shows the $3-D$ view of an Indian heritage object as displayed on the screen.

\subsection{Rendering at Different Scales}

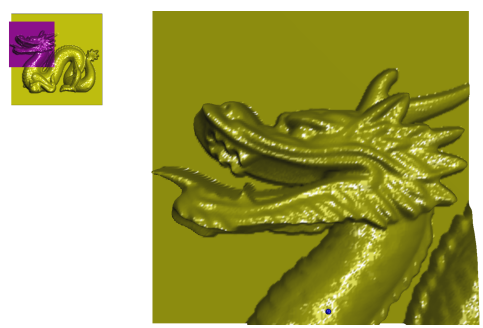

Fig. 4: Illustration of selection of a window for graphic and haptic rendering. (Data Courtesy:www.cc.gatech.edu/projects/large_models)

As mentioned earlier, heritage objects come at various physical scales- a few $\mathrm{cm}^{2}$ for coins and bas-reliefs to a several $\mathrm{km}^{2}$ for ancient ruins like Hampi. In a virtual museum, one should be able to experience objects of all sizes at different scales to get a sense of overall structure to a finer details from the same data set. Hence, we have implemented adaptive scaling in both graphic and haptic domains. In order to scale the surface we resize depth data of resolution $N \times N$ depending on the level we want, with $N \times N$ as the lowest level. If we load the level $N \times N$ into the haptic space the full object can be rendered visually as well as haptically. Users can select the level as well as the region of interest at run time either using buttons in the haptic device or using keyboard functions. Additionally, we have developed a graphical user interface for easy acessibility. The pink window in Fig. 4 represents the selected region to be zoomed in.

Depending on the scale selected by the user, only the corresponding depth data is dynamically loaded into the active haptic space and an appropriate haptic force is rendered. As only a limited subset of data is loaded, the rendering is very fast. In general, at higher levels of resolution, the user should be able to view higher depth value at each point and also more finer details. The haptic force also vary accordingly. Hence in order to incorporate realistic haptic and graphic perception, we need to appropriately scale 
the depth values at each level of depth map. Further, trying to map a large physical dimension over a small haptic work space (typically about 4 inch cube of active space) leads to a lot of unwanted vibrations (something similar in concept to aliasing) during rendering. Hence the depth values need to be smoothed before being downsampled and mapped into the haptic work space. The next section explains the generation of different levels of depth data.

\section{Multi-Scale Data Generation}

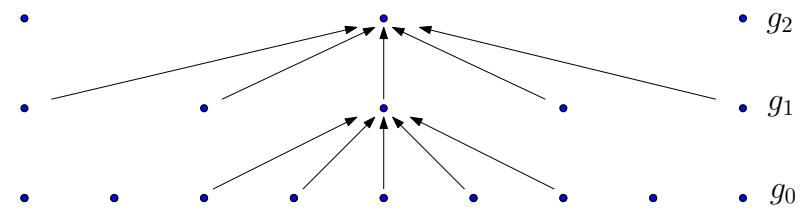

Fig. 5: A one-dimensional graphical representation of the process which generates a Gaussian pyramid. Each row of dots represents nodes within a level of the pyramid. The value of each node in the zero level is the magnitude of the corresponding depth map. The value of each node at a higher level is the decimated and weighted average of node values in the preceding level.

The aim of this work is to allow users to have access to the cultural heritage at different levels of details. To obtain the depth data at different levels of details, we perform Gaussian low-pass filtering followed by down sampling with a factor $M$ where $M$ can be any integer. We can also use fractional values of $M$, but it requires rational function approximation methods. In our work, we illustrate with $M=2$. For that the data pyramid offers a flexible and convenient multiresolution format that mirrors the different levels of details [5]. It consists of the available highest resolution depth data and a series of successively lower resolution data. Low-pass filtering before sub-sampling is done to prevent aliasing of data. Consequently, instability in the haptic domain is also prevented as smoothing removes higher frequency components responsible for micro textures on the surface. The presence of micro textures would have made sensing more realistic, but this makes the haptic rendering process miss to some extent a full understanding of the object at hand. The fine texture is experienced when the object is rendered at a finer scale by zooming into the object. The base, or zero level of the pyramid is equal to the original depth map $\left(g_{0}\right)$. Level 1 of the pyramid corresponds to depth map $g_{1}$ which is reduced or low-pass filtered version of $g_{0}$. Each value in level 1 is computed as a weighted average of values in level 0 within a $5 \times 5$ window. Each value in level $2\left(g_{2}\right)$ is then obtained from values of level 1 by applying the same pattern of weights. Fig. 5 shows the Gaussian pyramid of depth map. The depth value at each point at the level $l$ is given by the following equation:

$$
g_{l}(i, j)=\sum_{m=-2}^{2} \sum_{n=-2}^{2} w(m, n) g_{l-1}(2 i+m, 2 j+n),
$$


For levels $1<l<N+1$, and nodes $(i, j), 0<i<C_{l}, 0<j<R_{l}$, the upper level of the pyramid can be represented in the above given form. Here $N$ refers to the number of levels in the pyramid, while $C_{l}$ and $R_{l}$ are the dimensions at level $l$. The weighting pattern $w(m, n)$ is the Gaussian kernel [3].

\section{Results}

The proposed method was implemented in visual $\mathrm{C}++$ in a Windows XP platform with a CORE 2QUAD CPU @ 2.66 GHZ with 2 GB RAM. We have experimented with various models of cultural heritage objects and a few of them are displayed below. The Fig. 6 shows the model of Ganesh, visually rendered in OpenGL. For haptics rendering we use HAPI library. The blue ball represents the position of the proxy constrained to be on the surface. The discrete position in the model is displayed in a fixed $200 \times 200$ haptic space. The size and spatial resolution of the model depend on two factors: the active space of the haptic device used to render the model, and the resolution at which the model should be displayed. We use a 3-DOF haptic device from NOVINT with a 4 inch cube of active space. While interacting with the object haptically, the average proxy updation time is $0.0056 \mathrm{~ms}$ which is much less than the required upper bound of $1 \mathrm{~ms}$, and hence the user has very smooth haptic experience. The average time required for dynamic data generation and loading it into the haptic space depends on the resolution of input depth data and it was observed to be around $6.5 \mathrm{~s}$ and $2.0 \mathrm{~s}$ respectively for depth data with resolution of $800 \times 800$. As explained in the previous section, Fig. 6a corresponds to the lowest level of details. We also carry out the rendering at finer levels of details by successively zooming into the heritage object. These are shown in Fig. $6 \mathrm{~b}$ and Fig. $6 \mathrm{c}$

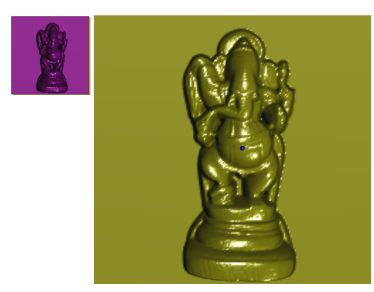

(a)

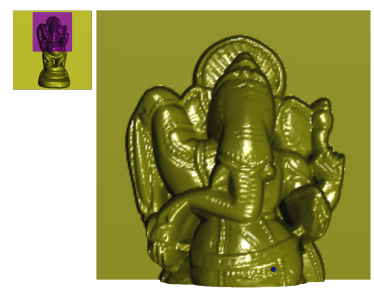

(b)

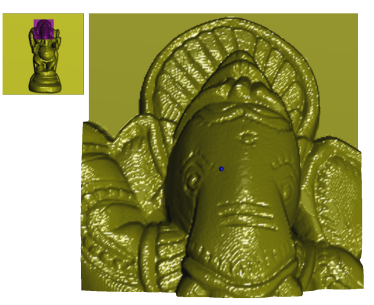

(c)

Fig. 6: Model of Ganesh, at (a) least level of details (b) at double the resolution and (c) at the finest resolution. (Data Courtesy: www.archibaseplanet.com)

In above cases, each figure consists of two parts where the left part is the reference for the users to select the part of the object they wish to explore haptically. The right part of the figure corresponds to the selected region at the appropriate resolution for haptic rendering. Fig. 6c shows the scaled up version of Fig. 6b It is quite clear from Fig. 6c that the users are able to feel even minute details of the sculpture and have visual 


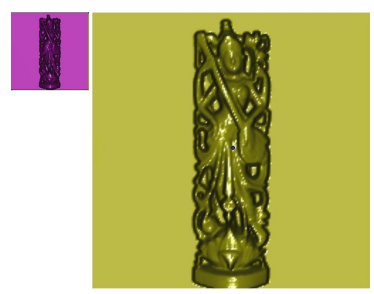

(a)

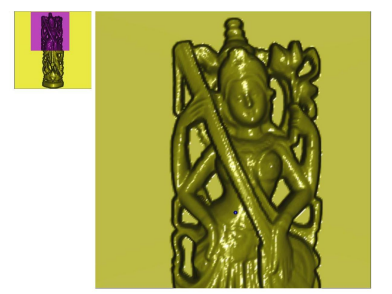

(b)

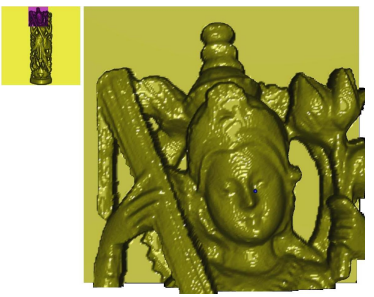

(c)

Fig. 7: Model of Goddess Saraswati at (a) level 1 (b) level 2 (c) level 3. (Data Courtesy: www.archibaseplanet.com)

perception of closeness in depth. Hence they can have a more realistic experience. The object rendered in Fig. 7 is a special case that illustrates how one can handle holes in the model. For any haptic rendering, holes in the model are difficult to accommodate as the proxy would sink through the hole and the user will perceive a wrong depth in the region around the holes. We avoid this by defining a base plane on which the object lies. Wherever, there is a hole, the depth at that point is replaced by $z(x, y)=z_{M A X}$ where $z_{M A X}$ is the maximum depth. This object has several holes, but the users reported a very good experience even in presence of such holes. Fig. $7 \mathrm{a}$ allows rendering at a coarser level while Fig. $7 \mathrm{~b}$ and Fig. $7 \mathrm{c}$ allow rendering at a much finer scale.

Validation of result is often a difficult task during haptic rendering, we demonstrate this using Fig. 8 that shows the reaction force versus time relation while haptically interacting with the depth data. The red line and the blue line in the figure shows the z-component of the HIP and the proxy point, respectively, during the interaction. The reaction force on the haptic device is also shown during the same time interval. In free space the HIP and proxy positions are almost the same as shown in part OA of the HIP position and hence the reaction force on the haptic device is zero. As the HIP penetrates the object the proxy stays on the surface according to the iteration method discussed in section 3 . The proxy point moves continuously during interaction, whenever there is a change in HIP position. This is shown with the part $A B$ in the curve. After the point $B$ the HIP position is kept constant inside the objects. As soon as the HIP is kept constant, the proxy quickly attains a stable position as shown in the Section BC in the curve. Fig. 8a shows the plots corresponding to the interaction on a flat region and Fig. 8b the same on a curved region, when a larger variation in force is observed.

In Fig. 9 we show the actual set up of our virtual haptic museum. A user wearing the anaglyphic glasses watches the stereoscopic visual rendering of the artefact and at the same time haptically interacts with the object with his hand. This provides an excellent hapto-visual immersion of the subject into the virtual object. However, for the visually impaired users, the selection of scale and the location for rendering cannot be based on the small navigation window on the screen. For such subjects, we use the buttons available on the haptic device for the user to explore the object at different scales and locations. 

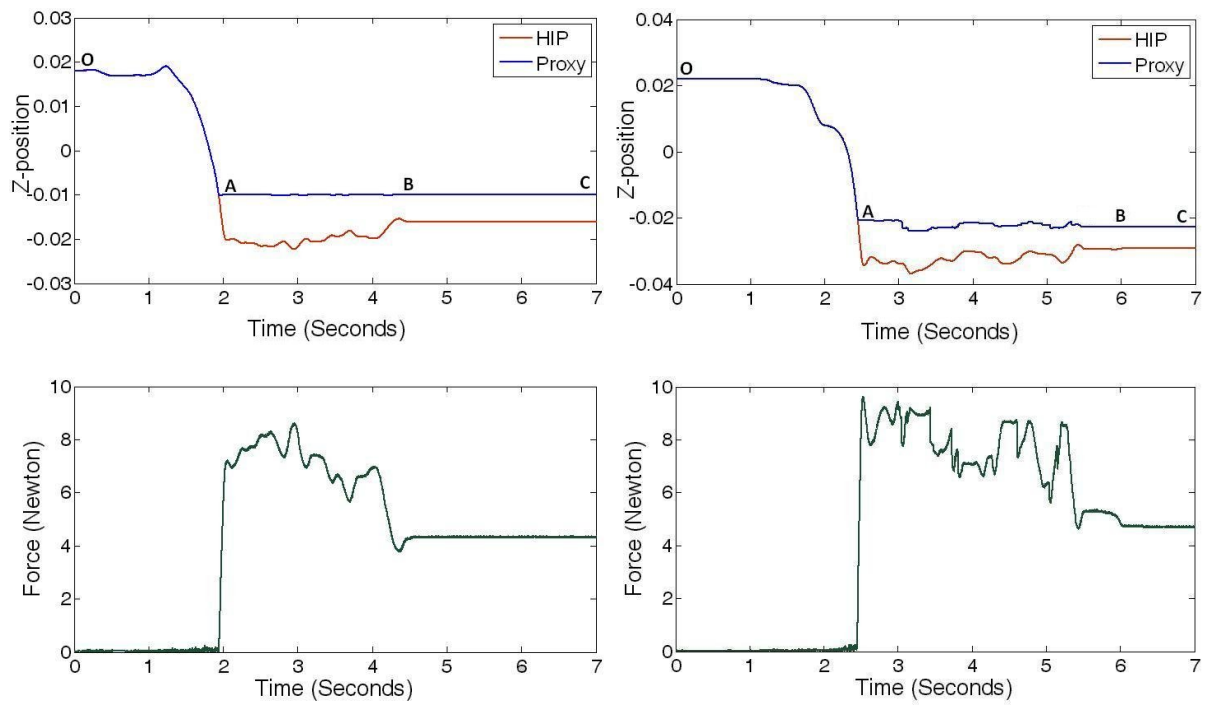

(a)

(b)

Fig. 8: Force vs. time graph for a particular interaction with the depth data a) on a flat region $\mathrm{b}$ ) on a curved region (The top figure correspond to changes in $\mathrm{z}$-coordinate only).

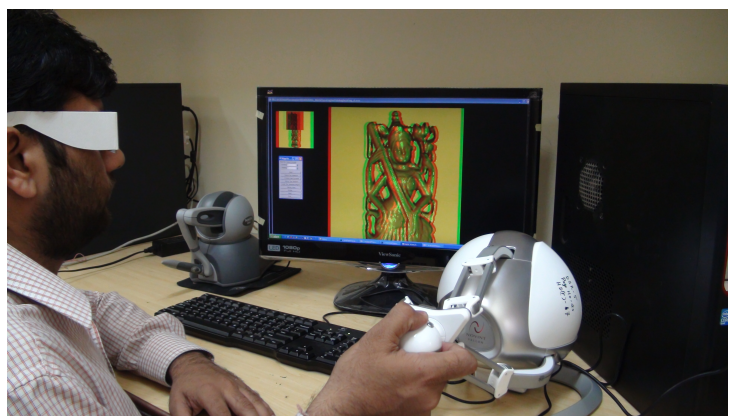

Fig. 9: Illustration of hapto-visual immersion of a subject for a virtual cultural heritage object. On the right, the user wearing anaglyphic glasses is holding the FALCON haptic device while interacting with the cultural heritage model displayed on the screen. 


\section{Conclusions}

In this work we have proposed a new technique of rendering cultural heritage objects represented as depth map data. Our primary goal is to provide access of cultural heritage objects and sites to the visually impaired people. Additionally, our method gives a better immersive experience to the sighted persons. We include scalability and stereoscopic display of $3-D$ models as additional features to enhance the realism in experience. We conducted experiments with several $3-D$ models of cultural significance. We also tested the rendering technique with some subjects and observed that hapto-visual rendering of virtual $3-D$ models using the proposed method greatly augmented the user's experience.

\section{References}

1. Ricardo S. Avila and Lisa M. Sobierajski. A haptic interaction method for volume visualization. Visualization Conference, IEEE, 0:197, 1996.

2. Massimo Bergamasco, Antonio Frisoli, and Federico Barbagli. Haptics technologies and cultural heritage applications. In IEEE Proceedings of the Computer Animation, CA '02, pages 25-, Washington, DC, USA, 2002. IEEE Computer Society.

3. P. Burt and E. Adelson. The laplacian pyramid as a compact image code. IEEE Transactions on Communications, 31(4):532 - 540, apr 1983.

4. Naim R. El-Far, Nicolas D. Georganas, and Abdulmotaleb El Saddik. An algorithm for haptically rendering objects described by point clouds. In Proceedings of the 21th Canadian Conference on Electrical and Computer Engineering, Niagara, ON, Canada, 2008.

5. Gluckman J. Scale variant image pyramids. In IEEE Computer Society Conference on Computer Vision and Pattern Recognition, 2006, volume 1, pages 1069 - 1075, june 2006.

6. Laehyun Kim, Anna Kyrikou, Mathieu Desbrun, and Gaurav Sukhatme. An implicit-based haptic rendering technique. In Proceeedings of the IEEE/RSJ International Conference on Intelligent Robots, 2002.

7. S. D. Laycock and A. M. Day. A survey of haptic rendering techniques. Computer Graphics Forum, 26(1):50-65, March 2007.

8. Jae-Kyu Lee and Young J. Kim. Haptic rendering of point set surfaces. World Haptics Conference, 0:513-518, 2007.

9. Adam Leeper, Sonny Chan, and Kenneth Salisbury. Constraint based 3-dof haptic rendering of arbitrary point cloud data. In RSS Workshop on RGB-D Cameras, University of Southern California, June 2011.

10. William A. Mcneely, Kevin D. Puterbaugh, and James J. Troy. Six degree-of-freedom haptic rendering using voxel sampling. In Proc. of ACM SIGGRAPH, pages 401-408, 1999.

11. Srinivasn M. A. Morgenbesser, H.B. Force shading for haptic shade perception. In Proceedings of the ASME Dynamic Systems and Control Division, volume 58, pages 407-412, 1996.

12. Matthias Renz, Carsten Preusche, Marco Pötke, Hans peter Kriegel, and Gerd Hirzinger. Stable haptic interaction with virtual environments using an adapted voxmap-pointshell algorithm. In Proc. Eurohaptics, pages 149-154, 2001.

13. Diego C. Ruspini, Krasimir Kolarov, and Oussama Khatib. The haptic display of complex graphical environments. In Proc. of ACM SIGGRAPH, pages 345-352, 1997.

14. Kenneth Salisbury, Francois Conti, and Federico Barbagli. Haptic rendering: Introductory concepts. IEEE Computer Graphics and Applications, 24(2):24-32, 2004. 
15. K. G. Sreeni and Subhasis Chaudhuri. Haptic Rendering of Dense 3D Point Cloud Data. In IEEE Haptic Symposium, Vancouver, BC, Canada, March 4-7 2012.

16. Cha Zhang and Jin Li. Interactive browsing of $3 \mathrm{~d}$ environment over the internet. In Proc. SPIE Visual Communications and Image Processing VCIP-2001, pages 509-520, 2001.

17. Craig B Zilles and J Kenneth Salisbury. A constraint-based god-object method for haptic display. In Proceedings 1995 ieee/rsj international conference on intelligent robots and systems. Human robot interaction and cooperative robots, volume 3, pages 146-151. IEEE, 1995. 